Draft version November 16, 2021

Typeset using LATEX twocolumn style in AASTeX63

\title{
Magnetar Models of Superluminous Supernovae from the Dark Energy Survey: Exploring Redshift Evolution
}

\author{
Brian Hsu (D), ${ }^{1}$ Griffin Hosseinzadeh (D) ${ }^{1}$ and Edo Berger (D) ${ }^{1}$ \\ ${ }^{1}$ Center for Astrophysics | Harvard \& Smithsonian, 60 Garden Street, Cambridge, MA 02138-1516, USA
}

\begin{abstract}
Superluminous supernovae (SLSNe) are luminous transients that can be detected to high redshifts with upcoming optical time-domain surveys such as the Vera C. Rubin Observatory Legacy Survey of Space and Time (LSST). An interesting open question is whether the properties of SLSNe evolve through cosmic time. To address this question, in this paper we model the multi-color light curves of all 21 Type I SLSNe from the Dark Energy Survey (DES) with a magnetar spin-down engine, implemented in the Modular Open-Source Fitter for Transients (MOSFiT). With redshifts up to $z \approx 2$, this sample includes some of the highest-redshift SLSNe. We find that the DES SLSNe span a similar range of ejecta and magnetar engine parameters to previous samples of mostly lower-redshift SLSNe (spin period $P \approx 0.79-13.61 \mathrm{~ms}$, magnetic field $B \approx(0.03-7.33) \times 10^{14} \mathrm{G}$, ejecta mass $M_{\mathrm{ej}} \approx 1.54-30.32$ $\mathrm{M}_{\odot}$, and ejecta velocity $\left.v_{\mathrm{ej}} \approx(0.55-1.45) \times 10^{4} \mathrm{~km} \mathrm{~s}^{-1}\right)$. The DES SLSN sample by itself exhibits the previously found negative correlation between $M_{\mathrm{ej}}$ and $P$, with a pronounced absence of SLSNe with low ejecta mass and rapid spin. Combining our results for the DES SLSNe with 60 previous SLSNe modeled in the same way, we find no evidence for redshift evolution in any of the key physical parameters.
\end{abstract}

Keywords: Supernovae (1668)

\section{INTRODUCTION}

Type I superluminous supernovae (hereafter, SLSNe) are a rare subclass of core-collapse supernovae (CCSNe) that have been discovered by wide-field time-domain optical surveys over the past decade (Chomiuk et al. 2011; Quimby et al. 2011). They were originally defined to have a peak absolute magnitude of $M<-21$ (Gal-Yam 2012), but are now defined spectroscopically by an absence of hydrogen features, blue continua, and, usually, unique early-time "W"-shaped O II absorption lines at $\sim 3600-4600 \AA$ (e.g. Lunnan et al. 2013; Mazzali et al. 2016; Quimby et al. 2018).

SLSNe radiate 10-100 times more energy in the UV/optical compared to normal CCSNe, and generally exhibit longer durations (e.g. Nicholl et al. 2015; Inserra et al. 2017; Lunnan et al. 2018; De Cia et al. 2018). As a result of their light curve behavior and spectral properties, it has become clear that SLSNe are not powered by radioactive decay of ${ }^{56} \mathrm{Ni}$ as in normal hydrogen-poor

Corresponding author: Brian Hsu

brianhsu@college.harvard.edu
CCSNe. Interaction with a hydrogen-poor circumstellar medium could in principle provide sufficient radiative energy, but there is no clear spectroscopic evidence for such interaction (Jerkstrand et al. 2017; Liu et al. 2017).

Instead, both the diverse light curves and the spectral evolution of SLSNe can be explained well with a central engine, namely a rapidly spinning $(\sim$ few ms) and highly magnetized $\left(\sim 10^{14} \mathrm{G}\right)$ neutron star (a "magnetar"; Kasen \& Bildsten 2010; Woosley 2010; Dessart et al. 2012; Metzger et al. 2015; Nicholl et al. 2017c). This model accounts for the broad range of peak luminosities and timescales (e.g., Nicholl et al. 2017c; Blanchard et al. 2020), for the early UV/optical spectra (e.g., Nicholl et al. 2017b), for the nebular phase spectra (e.g., Nicholl et al. 2016b, 2019; Jerkstrand et al. 2017), and, most recently, for the power law decline rate observed in SN 2015bn at $\sim 10^{3} \mathrm{~d}$ (Nicholl et al. 2018). Additional support for a magnetar engine comes from the low metallicity host galaxies of SLSNe, which most closely resemble the hosts of long gamma-ray bursts, another rare population of CCSNe that are powered by a central engine (e.g., Lunnan et al. 2014; Perley et al. 2016, but cf. Schulze et al. 2018). 
Systematic analyses of some SLSN samples in the context of a magnetar engine model have been carried out in recent years by Nicholl et al. (2017c), Villar et al. (2018), and Blanchard et al. (2020) using the semianalytical Modular Open-Source Fitter for Transients (MOSFiT; Guillochon et al. 2018). Recently, Angus et al. (2019) published a sample of 21 SLSNe at $z \approx 0.2-2$ discovered by the Dark Energy Survey (DES), presented their general observed properties, and derived physical properties from magnetar model fits to Gaussian process interpolated pseudo-bolometric light curves. Here, we use MOSFiT to model the multi-color light curves of these DES SLSNe with a magnetar engine for the first time to systematically compare them to the previouslypublished SLSN samples. Taking advantage of the broad redshift range of the DES sample, we also use the combined sample of 81 SLSNe to explore any redshift evolution in the engine and SN ejecta parameters.

The paper is structured as follows. The DES SLSN sample is summarized in $\S 2$. The model fitting procedure is described in $\S 3$. The resulting fits and model parameters are presented in $\S 4$, while in $\S 5$ we place these results in the context of previous SLSN samples analyzed in the same manner. Throughout the paper, we assume a flat $\Lambda \mathrm{CDM}$ cosmology with $\Omega_{\mathrm{m}}=0.308$ and $H_{0}=67.8 \mathrm{~km} \mathrm{~s}^{-1} \mathrm{Mpc}$, based on the Planck 2015 results (Planck Collaboration 2016).

\section{DATA SET}

We model the DES SLSN sample presented in Angus et al. (2019). DES (Dark Energy Survey Collaboration 2005, 2016) was an optical imaging survey covering 5000 $\mathrm{deg}^{2}$ of the southern sky, using the 570 megapixel Dark Energy Camera (DECam; Flaugher et al. 2015) on the 4-m Blanco Telescope at Cerro Tololo Inter-American Observatory in Chile. The DES-SN component of the survey (Bernstein et al. 2012), accounting for $\approx 10 \%$ of the total survey time, covered ten $3-\mathrm{deg}^{2}$ individual fields in $g, r, i, z$ with a roughly weekly cadence to typical limiting magnitudes of $\approx 23.5$ ( 8 fields) and $\approx 24.5$ (2 fields). See Kessler et al. (2015) for a more detailed description of the observing strategy, and Morganson et al. (2018) for the DES image processing pipeline.

Angus et al. (2019) classified transient events as SLSNe using SUPERFIT (Howell et al. 2005) with the SLSN spectral template library of Quimby et al. (2018). Their final sample consists of 21 SLSNe with a redshift range of $z=0.220-1.998$, separated into two classification "standards" of Gold (16 events) and Silver (5 events) based on their confidence in the spectroscopic classification, listed in Table 1. In the following analysis we correct the photometric measurements in Angus

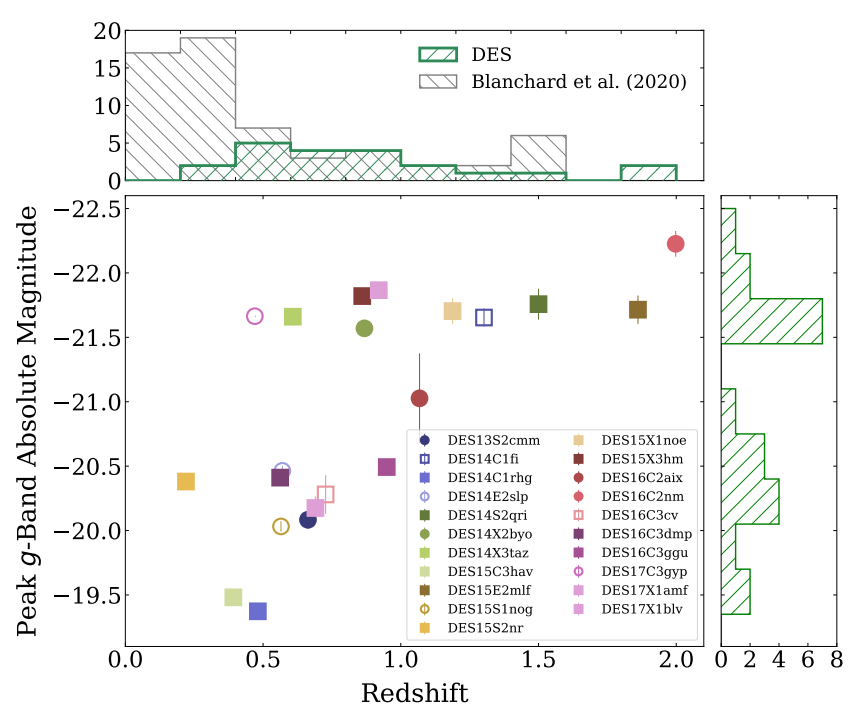

Figure 1. Rest-frame peak $g$-band absolute magnitude versus redshift for the DES SLSNe. The top panel shows the redshift distribution of the DES SLSNe (green) compared to the SLSN compilation recently analyzed by Blanchard et al. (2020). Square symbols indicate observed pre-peak bumps in the light curve via visual inspection. Solid and open markers indicate the Gold and Silver spectroscopic classification, respectively, of Angus et al. (2019).

et al. (2019) for Milky Way extinction using Schlafly \& Finkbeiner (2011).

In Figure 1 we plot the observed peak absolute magnitudes versus redshift for the DES sample, as well as the redshift distribution in comparison to the SLSN compilation from Blanchard et al. (2020). Because we do not have a complete set of spectra for the DES SLSNe, we correct our observed peak magnitudes to a single restframe filter by calculating synthetic photometry in the rest-frame $g$-band and the closest observed filter using the modified blackbody SED returned by MOSFiT (see $\S 3)$ at the epoch corresponding to the observed peak magnitude. See Table 1 for a complete list of redshift, observer-frame filter used, $K$ correction, and rest-frame peak $g$-band absolute magnitude for each DES SLSN.

The peak absolute magnitudes span a wide range of $\approx-19.5$ to $\approx-22.5$, with an apparent gap ${ }^{1}$ at about -20.5 to -21.5 . As expected, the lower luminosity events are mostly confined to lower redshift $(z \lesssim 1)$ while the more luminous events extend over $z \approx 0.5-2$. Angus et al. (2019) suspected that the

\footnotetext{
${ }^{1}$ There is one event, DES16C2aix, that has a peak rest-frame $g$ band absolute magnitude in this region. However, we note that the peak magnitude for DES16C2aix has large uncertainties and the next brightest point in rest-frame $g$-band resides on the lower bound of the plotted peak magnitude.
} 
Table 1. Peak Magnitudes of DES SLSNe in the Rest-frame $g$-band

\begin{tabular}{|c|c|c|c|c|c|c|c|c|c|}
\hline SLSN Name & Redshift & $\begin{array}{l}\text { Milky Way } \\
\text { Extinction } \\
E(B-V)\end{array}$ & $\begin{array}{c}\lambda_{\text {eff }} \text { of } \\
\text { Rest-frame } \\
g \text {-band }(\AA)\end{array}$ & $\begin{array}{l}\text { Closest } \\
\text { Observed } \\
\text { Band }\end{array}$ & $\begin{array}{l}\text { Observed } \\
\text { Peak } \\
\text { Mag. }\end{array}$ & $\begin{array}{c}\text { Peak Mag. } \\
\text { MJD } \\
(\mathrm{J} 2000)\end{array}$ & $K$ correction & $\begin{array}{c}\text { Rest-frame } \\
g \text {-band Peak } \\
\text { Abs. Mag. }\end{array}$ & Standard ${ }^{\dagger}$ \\
\hline DES13S2cmm & 0.663 & 0.0284 & 7934 & $i$ & 22.42 & 56567.18 & -0.56 & -20.08 & Gold \\
\hline DES14C1fi & 1.302 & 0.0091 & 10982 & $z$ & 22.09 & 56956.35 & -1.13 & -21.65 & Silver \\
\hline DES14C1rhg & 0.481 & 0.0096 & 7066 & $r$ & 22.30 & 57012.08 & -0.55 & -19.37 & Gold \\
\hline DES14E2slp & 0.57 & 0.0060 & 7490 & $i$ & 21.60 & 57042.09 & -0.61 & -20.46 & Silver \\
\hline DES14S2qri & 1.50 & 0.0276 & 11927 & $z$ & 22.31 & 57018.05 & -1.18 & -21.76 & Gold \\
\hline DES14X2byo & 0.868 & 0.0257 & 8912 & $z$ & 21.37 & 56956.21 & -0.84 & -21.57 & Gold \\
\hline DES14X3taz & 0.608 & 0.0220 & 7671 & $i$ & 20.60 & 57078.02 & -0.58 & -21.66 & Gold \\
\hline DES15C3hav & 0.392 & 0.0077 & 6641 & $r$ & 21.84 & 57339.14 & -0.38 & -19.48 & Gold \\
\hline DES15E2mlf & 1.861 & 0.0086 & 13649 & $z$ & 22.38 & 57396.05 & -1.73 & -21.71 & Gold \\
\hline DES15S1nog & 0.565 & 0.0541 & 7466 & $i$ & 22.08 & 57385.08 & -0.53 & -20.03 & Silver \\
\hline DES15S2nr & 0.220 & 0.0293 & 5820 & $r$ & 19.66 & 57319.13 & -0.21 & -20.38 & Gold \\
\hline DES15X1noe & 1.188 & 0.0177 & 10439 & $z$ & 21.88 & 57427.06 & -1.03 & -21.70 & Gold \\
\hline DES15X3hm & 0.860 & 0.0237 & 8874 & $z$ & 21.17 & 57249.36 & -0.77 & -21.82 & Gold \\
\hline DES16C2aix & 1.068 & 0.0114 & 9866 & $z$ & 22.51 & 57680.13 & -0.80 & -21.03 & Gold \\
\hline DES16C2nm & 1.998 & 0.0123 & 14303 & $z$ & 22.31 & 57627.40 & -1.47 & -22.23 & Gold \\
\hline DES16C3cv & 0.727 & 0.0103 & 8239 & $i$ & 22.54 & 57681.14 & -0.49 & -20.28 & Silver \\
\hline DES16C3dmp & 0.562 & 0.0068 & 7452 & $i$ & 21.66 & 57750.18 & -0.56 & -20.41 & Gold \\
\hline DES16C3ggu & 0.949 & 0.0074 & 9298 & $z$ & 22.38 & 57789.10 & -1.15 & -20.49 & Gold \\
\hline DES17C3gyp & 0.47 & 0.0073 & 7013 & $r$ & 19.96 & 58153.08 & -0.54 & -21.66 & Silver \\
\hline DES17X1amf & 0.92 & 0.0207 & 9160 & $z$ & 21.30 & 58076.13 & -0.77 & -21.87 & Gold \\
\hline DES17X1blv & 0.69 & 0.0213 & 8063 & $i$ & 22.39 & 58064.06 & -0.61 & -20.17 & Gold \\
\hline
\end{tabular}

$\dagger$ The confidence level in spectroscopic classification designated in Angus et al. (2019)

Note-All magnitudes have been corrected for Milky Way extinction using Schlafly \& Finkbeiner (2011). Absolute magnitudes are converted using Planck Collaboration 2016.

broad spread in peak magnitudes is due to varying injection times of magnetar power with respect to explosion times, where delayed injections cause systematically fainter peak magnitudes. The apparently bimodal peak magnitude distribution, with roughly equal numbers in each group, indicates that the lower luminosity events are intrinsically more common than the high luminosity events. In Section 5, we explore whether this bimodality is reflected in the physical parameters of the magnetar engine and/or ejecta.

In Figure 2 we show the full multi-band light curves of the 21 SLSNe. The light curves are well sampled and exhibit a wide range of morphologies in terms of rise and decline timescales. We also note that some light curves are smooth (e.g., DES15X3hm) while other exhibit eqaul-magnitude double peaks (e.g., DES16C3cv) or pronounced pre-peak "bumps" (e.g., DES15C3hav). A detailed discussion of the phenomenological light curve properties is provided by Angus et al. (2019).

\section{MAGNETAR ENGINE MODEL}

We fit the optical light curves of the 21 DES SLSNe using the Modular Open-Source Fitter for Transients (MOSFiT; Guillochon et al. 2018) with the magnetar spindown model described in Nicholl et al. (2017c). In short, MOSFiT is a open-source, Python-based light curve fitting package that employs a Markov chain Monte Carlo (MCMC) algorithm to fit analytical models to multiband light curves. Typically, blackbody SEDs can reproduce the optical and NIR broadband light curves of SLSNe (Nicholl et al. 2016a, 2017a) due to their relatively featureless spectra (Yan et al. 2017). However, it has been shown that the observed SED is subject to absorption at UV wavelengths (Mazzali et al. 2016; Quimby et al. 2018) that will dominate at observerframe optical wavelengths of higher redshift SLSNe, such as ones from DES. We therefore assume a modified SED where the overall shape follows a blackbody distribution but linearly suppressed blueward of $\approx 3000 \AA$ (see Fig- 
Hsu, Hosseinzadeh, \& Berger

ure 1 in Nicholl et al. 2017c). Nicholl et al. (2017c) also showed that this simplified SED assumption applies to a wide range of redshifts, and does not lead to systematic differences between the light-curve fits of higher and lower redshift SLSNe.

The magnetar model has 12 free parameters, of which 8 are nuisance parameters that we marginalize over to obtain the 4 physical parameters related to the ejecta and engine properties. We set one of the nuisance parameters, the angle $\theta_{P B}$ between the magnetic field and the rotational axis of the magnetar, to be constant at $90^{\circ}$. This choice ensures that the derived $B$-field strength is a lower limit (for more details, see Nicholl et al. 2017c). The nuisance parameters are not wellconstrained by the model. Events with sufficient latetime observations, however, are able to constrain the $\gamma$-ray opacity $\kappa_{\gamma}$. The neutron star mass is degenerate with the spin period and magnetic field strength but is not well constrained. The explosion time, $t_{\exp }$, is the time between explosion and first observation in the pure magnetar model. However, under the assumption that the pre-maximum bumps come from a different power source that we do not model, we can treat it as the time that the magnetar starts powering the light curve, rather than the true supernova explosion time. The main parameters that constrain the observed properties of a SLSN are the neutron star's initial spin period $P$, magnetic field strength $B$, ejecta mass $M_{\mathrm{ej}}$, and ejecta velocity $v_{\mathrm{ej}}$ (the latter two can be combined to determine the kinetic energy, $\left.E_{K}\right)$. The model parameters and their priors are listed in Table 2.

For each light curve fit, the first 10,000 iterations are used to burn in the ensemble, during which minimization is employed periodically as the ensemble converges to the global optimum; the remainder of the run-time is used to sample the posterior probability distribution. Convergence is measured by calculating the Gelman-Rubin statistic, or potential scale reduction factor (PSRF; Gelman \& Rubin 1992), which estimates the extent to which the full parameter space has been explored. We terminate our fits at $\operatorname{PSRF}<1.1$, which typically equates to $30,000-60,000$ iterations, depending on the the number of data points and the intrinsic scatter around our model.

As noted in $§ 2$, and seen in previous SLSNe (Nicholl \& Smartt 2016; Nicholl et al. 2017c; Lunnan et al. 2018; Angus et al. 2019), some of the DES events exhibit prepeak "bumps" in their light curves (Figure 2). These bumps were first recognized by Leloudas et al. (2012) in the light curve of SN $2006 \mathrm{oz}$ and have been interpreted in various ways (Piro 2015; Kasen et al. 2016; Margalit et al. 2018). However, since the MOSFiT magnetar en-
Table 2. Model Parameters and their Associated Priors

\begin{tabular}{llcccc}
\hline \multicolumn{1}{c}{ Parameter } & Prior & Min & Max & Mean & S.D. \\
\hline$P / \mathrm{ms}$ & Flat & 0.7 & 10 & $\ldots$ & $\ldots$ \\
$B / 10^{14} \mathrm{G}$ & Flat & 0.1 & 10 & $\ldots$ & $\ldots$ \\
$M_{\mathrm{ej}} / \mathrm{M}_{\odot}$ & Flat & 0.1 & 100 & $\ldots$ & $\ldots$ \\
$\nu_{\mathrm{ej}} / 10^{4} \mathrm{~km} \mathrm{~s}^{-1}$ & Gaussian & 0.1 & 3.0 & 1.47 & 4.3 \\
$\kappa / \mathrm{g} \mathrm{cm}^{-2}$ & Flat & 0.05 & 0.2 & $\ldots$ & $\ldots$ \\
$\kappa_{\gamma} / \mathrm{g} \mathrm{cm}^{-2}$ & Log-flat & 0.01 & 100 & $\ldots$ & $\ldots$ \\
$M_{\mathrm{NS}} / \mathrm{M}_{\odot}$ & Flat & 1.4 & 2.2 & $\ldots$ & $\ldots$ \\
$T_{\mathrm{min}} / 10^{3} \mathrm{~K}$ & Gaussian & 3.0 & 10.0 & 6.0 & 1.0 \\
$n_{\mathrm{H}, \text { host }}$ & Log-flat & $10^{16}$ & $10^{23}$ & $\ldots$ & $\ldots$ \\
$t_{\mathrm{exp}} /$ days & Flat & -100 & 0 & $\ldots$ & $\ldots$ \\
$\sigma$ & Log-flat & $10^{-3}$ & 100 & $\ldots$ & $\ldots$ \\
\hline
\end{tabular}

Note $-P$ is the initial spin period of the magnetar; $B$ is the magnetic field strength; $M_{\mathrm{ej}}$ is the ejecta mass; $v_{\mathrm{ej}}$ is the ejecta velocity; $\kappa$ is the opacity; $\kappa_{\gamma}$ is the gammaray opacity; $M_{\mathrm{NS}}$ is the neutron star mass; $T_{\min }$ is the photospheric temperature floor; $n_{\mathrm{H} \text {,host }}$ is the hydrogen number density of the host galaxy; $t_{\exp }$ characterizes the time of explosion before the first observed data point; $\sigma$ is the additional fractional uncertainty required to yield a reduced $\chi^{2}$ of 1 . For a detailed description of the model see Nicholl et al. (2017c).

gine model does not account for these bumps, we exclude these time ranges from our fits via visual inspection (Figure 2). Whereas Angus et al. (2019) looked specifically for DES14X3taz-like and DES15S2nr-like bumps in the rest-frame $g$-band light curves, we exclude any unusual detection prior to the main peak in any band. In practice, this only affects a third of the events, and eliminates a small fraction of the data for these events.

Finally, to place the DES SLSN sample in context, and to investigate any redshift evolution of the physical parameters, we compare our results to a sample of 60 SLSNe from other surveys previously modeled in the same manner (Nicholl et al. 2017c; Villar et al. 2018; Blanchard et al. 2018, 2019, 2020); see Blanchard et al. (2020) for the full list of events and their references. Taken together, the DES and literature SLSN samples span a wide range of properties and redshifts, $z \approx 0.057-1.998$ (see top panel in Figure 1).

\section{LIGHT CURVE FITS}

In Figure 2 we show the magnetar model light curve fits. The posterior probability distributions for each parameter consist of the last 5000 steps in each of the 120 Markov chains. We list the parameter median values and $1 \sigma$ uncertainties in Table 3, where the lower and upper error bars correspond to the 16th and 84th per- 

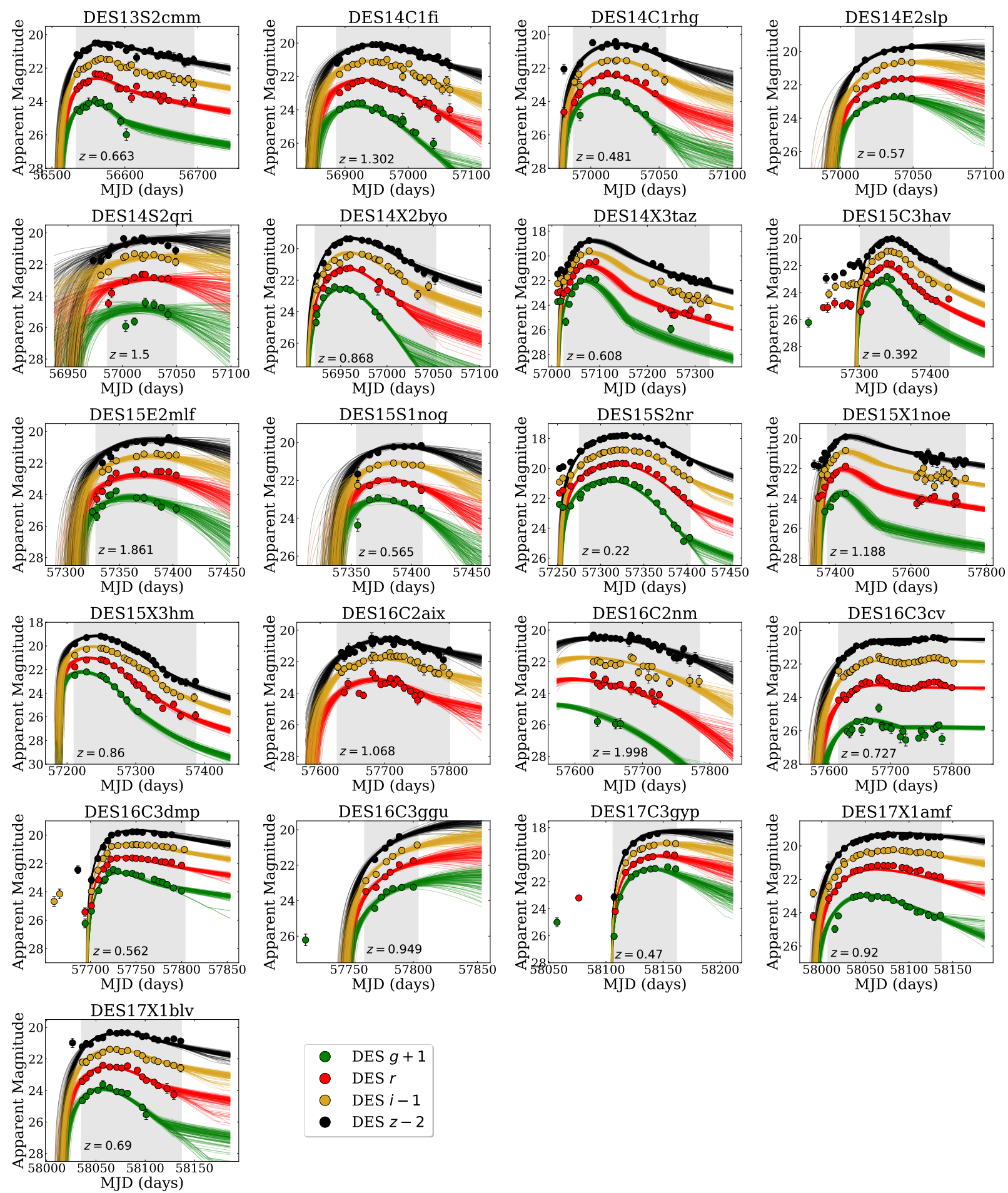

Figure 2. Multi-band extinction-corrected apparent magnitude light curves of the 21 DES SLSNe along with our magnetar model fits using MOSFiT. The different filters are shifted for clarity, as indicated in the legend. The grey shaded regions mark the time ranges used in the fitting, designed to remove significant pre-peak bumps. 
Table 3. Median and $1 \sigma$ Values for Key Magnetar Engine Model Parameters

\begin{tabular}{|c|c|c|c|c|c|c|c|c|}
\hline SLSN Name & $\begin{array}{c}P \\
(\mathrm{~ms})\end{array}$ & & $\begin{array}{r}B \\
\left(10^{14}\right.\end{array}$ & G) & $\begin{array}{c}M_{\mathrm{ej}} \\
\left(\mathrm{M}_{\odot}\right)\end{array}$ & & $\left(10^{3} \mathrm{~km} \mathrm{~s}^{v_{\mathrm{ej}}}\right)$ & $\begin{array}{c}\sigma \\
(\mathrm{mag})\end{array}$ \\
\hline DES13S2cmm & $9.25_{-1.09}^{+0.80}$ & & $1.90_{-0.42}^{+0.36}$ & & $2.63_{-0.64}^{+1.01}$ & & $8.29_{-0.64}^{+0.67}$ & $0.13_{-0.02}^{+0.02}$ \\
\hline DES14C1fi & $3.27_{-0.42}^{+0.33}$ & (3.64) & $0.94_{-0.24}^{+0.21}$ & $(3.12)$ & $4.87_{-1.35}^{+1.91}$ & $(17.32)$ & $7.83_{-0.57}^{+0.62}$ & $0.16_{-0.02}^{+0.02}$ \\
\hline DES14C1rhg* & $13.33_{-2.03}^{+1.83}$ & $(4.95)$ & $7.33_{-1.86}^{+1.61}$ & (6.03) & $2.31_{-0.95}^{+1.43}$ & $(3.19)$ & $6.26_{-0.44}^{+0.46}$ & $0.12_{-0.02}^{+0.03}$ \\
\hline DES14E2slp & $6.53_{-1.00}^{+0.91}$ & & $1.69_{-0.64}^{+0.89}$ & & $3.47_{-1.29}^{+1.98}$ & & $7.26_{-0.76}^{+0.78}$ & $0.11_{-0.03}^{+0.03}$ \\
\hline DES14S2qri* & $2.09_{-0.73}^{+0.96}$ & $(4.95)$ & $0.25_{-0.15}^{+0.32}$ & $(6.03)$ & $4.56_{-1.77}^{+7.39}$ & $(3.19)$ & $12.30_{-3.98}^{+3.20}$ & $0.27_{-0.05}^{+0.06}$ \\
\hline DES14X2byo & $3.18_{-0.55}^{+0.74}$ & $(5.88)$ & $0.59_{-0.20}^{+0.36}$ & $(4.99)$ & $2.14_{-0.34}^{+0.35}$ & $(3.19)$ & $14.50_{-0.72}^{+0.76}$ & $0.15_{-0.02}^{+0.02}$ \\
\hline DES14X3taz* & $3.01_{-0.45}^{+0.45}$ & & $0.44_{-0.12}^{+0.15}$ & & $4.62_{-0.69}^{+0.70}$ & & $10.93_{-0.81}^{+0.84}$ & $0.24_{-0.03}^{+0.03}$ \\
\hline DES15C3hav* & $13.61_{-1.65}^{+1.30}$ & & $4.35_{-1.12}^{+1.30}$ & & $2.12_{-0.45}^{+0.59}$ & & $6.80_{-0.50}^{+0.56}$ & $0.23_{-0.02}^{+0.03}$ \\
\hline DES15E2mlf* & $2.73_{-1.03}^{+0.61}$ & $(5.60)$ & $1.58_{-0.54}^{+0.70}$ & $(2.68)$ & $4.17_{-1.88}^{+4.94}$ & $(1.10)$ & $8.62_{-1.33}^{+1.51}$ & $0.26_{-0.04}^{+0.05}$ \\
\hline DES15S1nog & $7.17_{-3.36}^{+1.63}$ & & $3.91_{-1.50}^{+2.48}$ & & $3.90_{-2.04}^{+3.76}$ & & $5.47_{-0.69}^{+0.82}$ & $0.12_{-0.03}^{+0.03}$ \\
\hline DES15S2nr* & $5.93_{-0.74}^{+0.63}$ & $(10.52)$ & $2.37_{-0.55}^{+0.54}$ & $(12.47)$ & $7.52_{-0.86}^{+0.96}$ & $(6.17)$ & $6.40_{-0.21}^{+0.21}$ & $0.13_{-0.01}^{+0.01}$ \\
\hline DES15X1noe* & $3.34_{-0.40}^{+0.30}$ & $(7.45)$ & $0.62_{-0.14}^{+0.12}$ & $(2.36)$ & $2.75_{-0.59}^{+0.90}$ & $(1.15)$ & $12.55_{-1.59}^{+1.47}$ & $0.16_{-0.04}^{+0.04}$ \\
\hline DES15X3hm & $1.08_{-0.25}^{+0.33}$ & $(5.63)$ & $3.31_{-0.79}^{+0.78}$ & $(3.49)$ & $10.06_{-1.86}^{+3.91}$ & $(4.18)$ & $13.93_{-1.06}^{+1.20}$ & $0.14_{-0.01}^{+0.02}$ \\
\hline DES16C2aix & $2.57_{-0.80}^{+0.97}$ & & $0.18_{-0.10}^{+0.18}$ & & $7.19_{-1.74}^{+1.96}$ & & $9.22_{-0.99}^{+1.02}$ & $0.17_{-0.03}^{+0.03}$ \\
\hline DES16C2nm & $0.80_{-0.07}^{+0.15}$ & & $1.78_{-0.34}^{+0.23}$ & & $30.32_{-5.76}^{+7.89}$ & & $12.84_{-1.06}^{+1.13}$ & $0.15_{-0.03}^{+0.03}$ \\
\hline DES16C3cv & $1.52_{-0.41}^{+0.63}$ & & $0.03_{-0.02}^{+0.04}$ & & $9.95_{-2.96}^{+5.37}$ & & $9.44_{-0.69}^{+0.72}$ & $0.14_{-0.01}^{+0.01}$ \\
\hline DES16C3dmp* & $7.04_{-0.88}^{+0.60}$ & $(18.27)$ & $1.37_{-0.32}^{+0.28}$ & $(9.98)$ & $1.54_{-0.56}^{+0.71}$ & $(0.23)$ & $8.77_{-0.49}^{+0.50}$ & $0.15_{-0.01}^{+0.02}$ \\
\hline DES16C3ggu* & $2.06_{-0.88}^{+1.21}$ & $(4.80)$ & $0.74_{-0.35}^{+0.50}$ & $(1.05)$ & $15.80_{-9.60}^{+29.73}$ & $(16.24)$ & $6.05_{-0.83}^{+0.82}$ & $0.14_{-0.02}^{+0.03}$ \\
\hline DES17C3gyp* & $4.30_{-0.57}^{+0.50}$ & $(6.29)$ & $1.07_{-0.33}^{+0.41}$ & $(2.94)$ & $1.98_{-0.44}^{+0.60}$ & $(11.57)$ & $12.42_{-1.01}^{+0.98}$ & $0.19_{-0.03}^{+0.03}$ \\
\hline DES17X1amf* & $2.58_{-0.33}^{+0.31}$ & $(5.56)$ & $0.30_{-0.08}^{+0.09}$ & $(2.22)$ & $3.28_{-0.52}^{+0.59}$ & (11.39) & $13.36_{-0.68}^{+0.75}$ & $0.16_{-0.01}^{+0.03}$ \\
\hline DES17X1blv* & $8.98_{-1.07}^{+0.78}$ & & $2.16_{-0.50}^{+0.46}$ & & $2.42_{-0.50}^{+0.68}$ & & $9.62_{-0.61}^{+0.63}$ & $0.10_{-0.02}^{+0.02}$ \\
\hline
\end{tabular}

*SLSNe showing evidence for light curve pre-peak "bumps"

Note-Values quoted in parentheses are the derived physical properties from the magnetar model fits in Angus et al. (2019), where available. 
centiles of the posterior, respectively. We find that most fits are excellent and well converged, and the model parameters are well determined. The SN colors are well matched, with no apparent systematic offsets. We also include in Table 3 results from Angus et al. (2019). There are several discrepancies in the $P, B$, and $M_{\mathrm{ej}}$ values outputted by MOSFiT and those reported by Angus et al. (2019), which we suspect are caused by different methods used to fit the data (i.e., fitting the pseudobolometric light curves vs. multi-band light curves).

Our model includes an intrinsic scatter term, $\sigma$, that when uniformly added to all data points results in a reduced $\chi^{2}$ of 1 ; this parameter provides insight into the quality of the fits. We find that the median value for the DES SLSN sample is $\sigma \approx 0.15 \mathrm{mag}$, about a factor of 2 higher than the reported photometric errors on individual data points. This indicates some additional scatter in the data compared to the smooth magnetar models, but an overall reasonable match to the data. The main discrepancies appear to be small amplitude "wiggles" in the light curves that are not captured in the model, and which have been seen in previous SLSNe (e.g., Nicholl et al. 2016a).

The model posteriors are much narrower than our priors, implying that the priors do not significantly affect our results. The DES SLSN sample median values, and associated $1 \sigma$ ranges, of the four key physical parameters, along with the ejecta kinetic energy ${ }^{2}$, $E_{K}=\frac{1}{2} M_{\mathrm{ej}} v_{\mathrm{ej}}{ }^{2}$, are listed in Table 4 . We also list in the table the values for the SLSN compilation from Blanchard et al. (2020). Overall, the model parameters for the DES and literature samples are in good agreement within the $1 \sigma$ ranges. In $\S 5$ we examine in more detail the derived parameters, any correlations between them, and any redshift evolution.

\section{ANALYSIS}

With the higher redshift range spanned by DES sample, we explore any redshift evolution in the SLSN parameters, or correlations between them. Any redshift evolution would point to evolution in the properties of the progenitors or the magnetar engines over cosmic time, potentially reflecting evolution in the SLSN environments (e.g., metallicity, star formation activity).

\subsection{Bolometric Light Curve Properties}

We use the MOSFiT models to generate bolometric light curves for the DES SLSNe, from which we measure the

\footnotetext{
${ }^{2}$ Our model assumes the analytic density profile described in Margalit et al. (2018). For a homogeneous density profile, the kinetic energy is given by $E_{K}=\frac{3}{10} M_{\mathrm{ej}} v_{\mathrm{ej}}^{2}$.
}

Table 4. DES SLSN Sample Median Values

\begin{tabular}{lcc}
\hline \hline \multicolumn{1}{c}{ Parameter } & DES & Blanchard et al. (2020) \\
\hline$P(\mathrm{~ms})$ & $3.27_{-1.20}^{+5.35}$ & $2.35_{-1.06}^{+2.15}$ \\
$B\left(10^{14} \mathrm{G}\right)$ & $1.37_{-1.05}^{+1.75}$ & $0.98_{-0.77}^{+1.77}$ \\
$M_{\mathrm{ej}}\left(\mathrm{M}_{\odot}\right)$ & $3.90_{-1.72}^{+5.57}$ & $4.45_{-2.31}^{+5.23}$ \\
$v_{\mathrm{ej}}\left(10^{3} \mathrm{~km} \mathrm{~s}^{-1}\right)$ & $9.22_{-2.75}^{+3.56}$ & $9.38_{-2.72}^{+3.17}$ \\
$E_{K}\left(10^{51} \mathrm{erg}\right)$ & $3.08_{-1.78}^{+3.62}$ & $3.78_{-1.71}^{+5.07}$ \\
\hline
\end{tabular}

Note-The median values and $1 \sigma$ ranges for the magnetar engine and ejecta parameters of the DES SLSN sample and the SLSN compilation sample from Blanchard et al. (2020).

peak luminosities, durations (exponential rise and decline times), and radiated energies. Instead of calculating pseudo-bolometric light curves by summing the observed griz photometry, MOSFiT constructs true bolometric light curves before any SED is applied.

In Figure 3 we show the resulting peak bolometric luminosities $\left(L_{\mathrm{p}, \mathrm{bol}}\right)$ and radiated energies $\left(E_{\mathrm{rad}}\right)$ versus redshift. In order to compare events with different observed phase ranges, we calculate $E_{\text {rad }}$ by integrating the bolometric model from the explosion time, $t_{\exp }$, to $3 e$-folding times after peak, extrapolating the model where necessary. In principle, this should account for the majority of total energy radiated by each SLSN. We find a range of $L_{\mathrm{p} \text {,bol }} \approx(0.22-4.99) \times 10^{44} \mathrm{erg} \mathrm{s}^{-1}$, and continued evidence for an apparent bimodal distribution with a gap at $\approx(1-2) \times 10^{44} \mathrm{erg} \mathrm{s}^{-1}$, as in the case of peak absolute $g$-band magnitudes (§2). For $E_{\text {rad }}$, which reflects both the luminosity and the duration of each event, we find a range of $\approx(0.09-3.67) \times 10^{51} \mathrm{erg}$ and a less pronounced bimodality. This may be due to the distribution of light curve durations, which we show as the combined exponential rise and decline time in Figure 4; we define these as the timescales to brighten from and $\operatorname{dim}$ to $L=L_{\mathrm{p}, \text { bol }} / e$. We find a wide spread in timescale as a function of $L_{\mathrm{p}, \mathrm{bol}}$, which acts to smooth out the distribution of $E_{\mathrm{rad}}$. We exclude DES16C3cv from the right panel of Figure 3 and Figure 4 because its model light curve, affected by the roughly equal-magnitude peaks in the data, does not decline by a factor of $e$ within a reasonable time frame.

\subsection{Physical Properties and Correlations}

In Figure 5 we show two-dimensional distributions of the primary physical parameters $\left(P, B, M_{\mathrm{ej}}\right.$, and $\left.v_{\mathrm{e}}\right)$ and the redshifts of the DES SLSNe and the comparison sample from Blanchard et al. (2020). We explore both differences between the two samples, and param- 

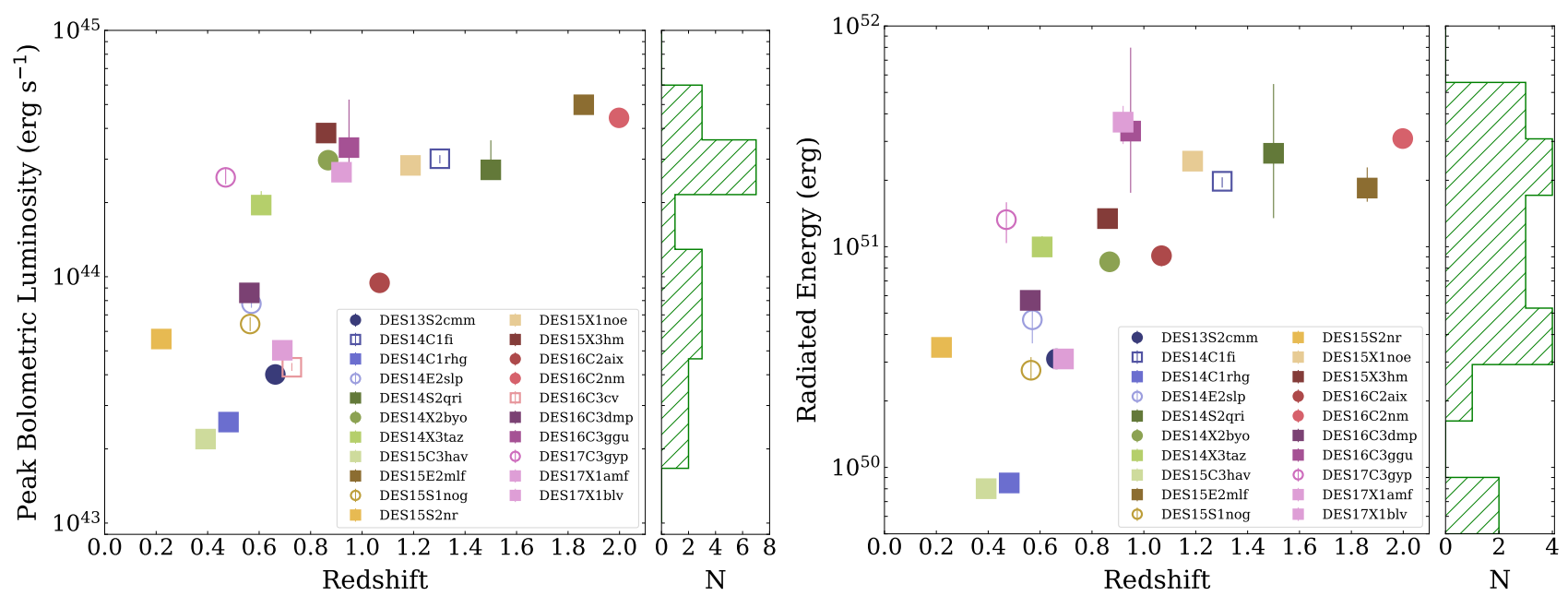

Figure 3. Peak bolometric luminosities (Left) and radiated energies (Right) plotted as functions of redshift. The values are calculated from the MOSFiT magnetar engine models. We still find evidence for a bimodality in the peak bolometric luminosities, but a less pronounced one in the total radiated energies. The radiated energies plotted here are lower limits, obtained by integrating each bolometric light curve from the explosion time, $t_{\exp }$ to $3 e$-folding times after peak. Square symbols indicate observed pre-peak bumps in the light curve via visual inspection. Solid and open markers indicate the Gold and Silver spectroscopic classification, respectively, of Angus et al. (2019).

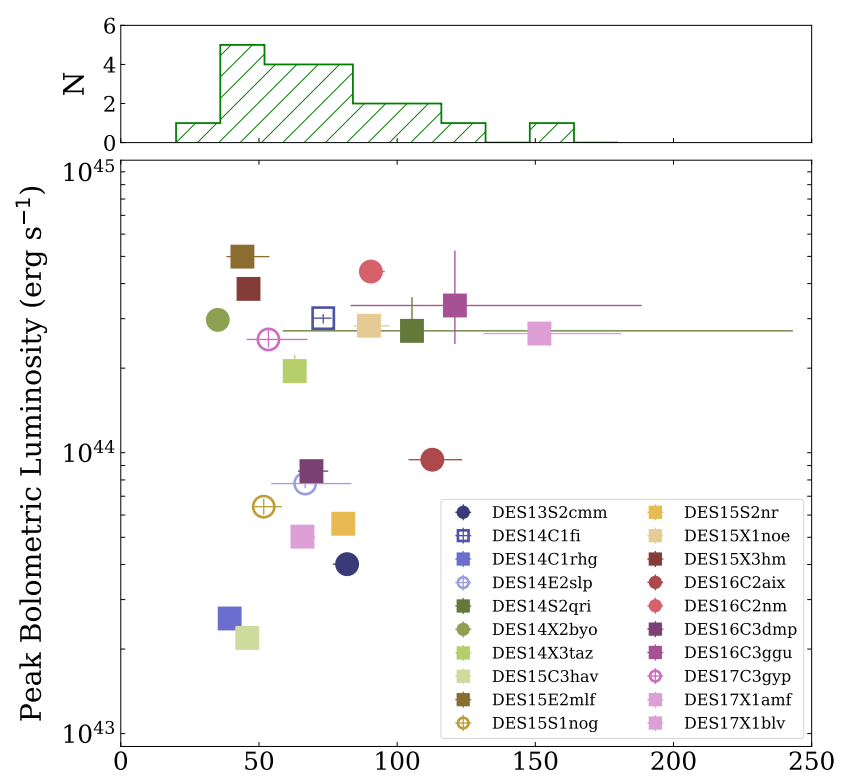

Exponential Rise + Decline Time (Rest Frame Days)

Figure 4. Peak bolometric luminosity versus exponential timescale measured from the model bolometric light curves. Square symbols indicate observed pre-peak bumps in the light curve via visual inspection. Solid and open markers indicate the Gold and Silver spectroscopic classification, respectively, of Angus et al. (2019).

eter correlations for the combined sample. Specifically, we compare the DES and literature samples using the two-sample Kolmogorov-Smirnov (K-S) test, and use the resulting $p$-values to determine if both are drawn from the same underlying distribution. The values are quoted in Figure 5. We find that the only parameter in which the DES and literature samples differ significantly is the redshift. This is due to the nature of the DES survey (deep pencil beam) compared to for example PTF (shallow wide field). In terms of the magnetar model parameters we find that the distirbutions are in good agreement; the most apparent difference is in $P$ (with $p \approx 0.1$ ), but this is not a statistically significant result. Thus, we conclude that the DES SLSNe are similar to those found in other surveys, and simply tend to be at somewhat higher redshifts.

With this in mind, we combine the DES and literature samples to explore parameters correlations, including correlations with redshift. For each pair of parameters, we take uncertainties into account and perform a Monte Carlo procedure to calculate the Spearman rank correlation coefficient ( $\rho$; Spearman 1904) and its associated $1 \sigma$ bound using the method described in Curran (2014). For the model parameters, we find that most combinations exhibit either no correlation (e.g., $M_{\mathrm{ej}}$ versus $v_{\mathrm{ej}}$ ) or mild correlations that are primarily due to the absence of events in specific subsets of the parameter space. For example, we find $\rho \approx 0.32$ for $P$ versus $B$, which is due primarily to the absence of observed events in the slow spin and low $B$-field portion of the parameter space. This is likely an observational bias, which we investigate in $\S 5.3$. The strongest correlation we find is $M_{\mathrm{ej}}$ versus $P$ with $\rho \approx-0.55$. This correlation was noted and discussed in Blanchard et al. (2020). We see no obvious bimodality in the distribution of any physical parameter. 


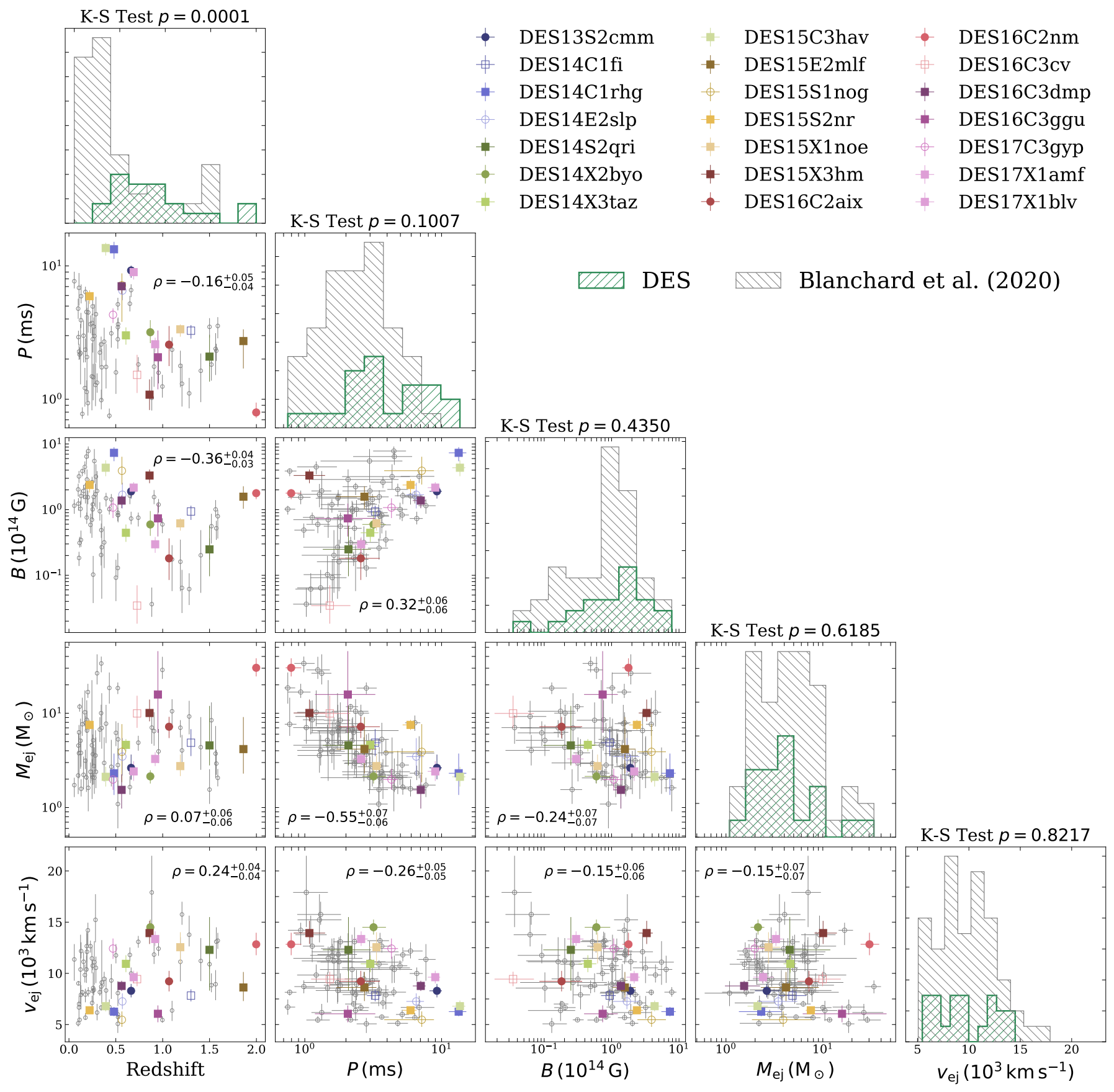

Figure 5. Median values and $1 \sigma$ uncertainties of the key model parameters $\left(P, B, M_{\mathrm{ej}}, v_{\mathrm{ej}}\right)$. The gray points mark events from Blanchard et al. (2020) while the colored points are DES SLSNe modeled in this paper. In the top panels we compare the parameter distributions for the DES (green) and literature (grey) samples, along with the $p$-value associated with the K-S test statistic. Besides redshift, we conclude that the physical parameters for both samples are drawn from the same distribution. In each panel we quote the median value and $1 \sigma$ bound of the Spearman rank correlation coefficient using the combined DES and literature data set. Of all pairs, $P$ and $M_{\mathrm{ej}}$ exhibit the strongest correlation, consistent with the finding in Blanchard et al. (2020). 
In terms of redshift evolution, we find that none of the physical parameters appear to be strongly correlated with redshift $(|\rho| \lesssim 0.36$ for all parameters). We therefore conclude that at least to $z \approx 2$ (look-back time of $\approx 10.4 \mathrm{Gyr}$ ) there is no evidence for redshift evolution in the properties of the engine and/or ejecta in SLSNe.

\subsection{The Effect of Observational Biases}

Certain combinations of the magnetar engine and/or ejecta parameters lead to fainter light curves that are less easily detectable by observational surveys. Such an effect might lead to apparent correlations in the model parameters that are driven by observational bias rather than an actual physical effect. To address this possibility we use a grid of 3,000 simulated SLSN light curves from Blanchard et al. (2020), sampled from uniform prior distributions of $P=1-8 \mathrm{~ms}, B=(0.03-10) \times 10^{14} \mathrm{G}$, $M_{\mathrm{ej}}=1-60 \mathrm{M}_{\odot}$, and a Gaussian distribution of $v_{\mathrm{ej}}$ with a mean of $1.47 \times 10^{4} \mathrm{~km} \mathrm{~s}^{-1}$ and a standard deviation of $4.3 \times 10^{4} \mathrm{~km} \mathrm{~s}^{-1}$ (based on values reported by Liu et al. 2017).

For each simulated light curve, we convert the resulting rest-frame $r$-band absolute magnitudes to apparent magnitudes at $z=0.01-2$ in steps of $\Delta z=0.01$. Each of the resulting 600,000 light curves has a unique set of parameters $\left(z, P, B, M_{\mathrm{ej}}\right.$, and $\left.v_{\mathrm{ej}}\right)$, which we then treat as an individual SLSN. In reality, the processes in which optical time-domain surveys (including DES) identify SLSNe are more complicated and often require spectroscopy. We focus here solely on whether we can observe any portion of a light curve theoretically.

In Figure 6, we show the resulting two-dimensional density plots for each pair of parameters. The plot is structured in the same fashion as Figure 5 to allow a direct comparison. The $r$-band peak magnitude assigned to each hexagonal cell is the mean of all model SLSNe that reside in the cell. We find that several of the general trends observed in the data are observed in this plot, and can therefore be attributed to observational biases. For example, the dearth of observed SLSNe with slow $P$ at high redshift (top left panel of Figure 5) is due to the faintness of such events, with peak apparent magnitudes of $\gtrsim 24$. Similarly, the absence of events with slow spin and low values of $B$ (Figure 5 ) is again seen as a region of peak apparent magnitudes of $\gtrsim 24$ in Figure 6 .

On the other hand, the strong negative correlation between $M_{\mathrm{ej}}$ and $P$ cannot be explained by an observational bias. The upper right corner of this parameter space (high $M_{\mathrm{ej}}$ and slow $P$ ) indeed tends to lead to fainter SLSNe, but the opposite combination (low $M_{\mathrm{ej}}$ and fast $P$ ) leads to bright events that should otherwise be detectable in all surveys. Thus, as noted by
Blanchard et al. (2020), the absence of SLSNe with this combination of physical parameters seems to point to a physical origin.

\section{CONCLUSIONS}

We carried out the first systematic modeling of the 21 SLSNe discovered in DES using the magnetar engine model implemented in MOSFiT, building on our previous work of modeling SLSNe from other surveys. While the DES sample exhibits a wide range of light curve luminosities and shapes, the sample can be well explained with the magnetar model. We find significant overlap between the model parameter distributions of the DES sample and previous SLSNe from various surveys (evaluated with a K-S test), despite the overall higher redshift of the DES sample.

Examining possible correlations between the model physical parameters $\left(P, B, M_{\mathrm{ej}}\right.$, and $\left.v_{\mathrm{ej}}\right)$ for the combined sample of about 80 SLSNe we recover the strong negative correlation between $M_{\mathrm{ej}}$ and $P$ previously found by Blanchard et al. (2020). We further find regions devoid of observed SLSNe in some of the twodimensional parameter spaces. However, by generating a grid of models across a wide redshift range we find that these regions of parameter space lead to dim events below the detection limit of DES and other surveys.

Using the combined SLSN sample, and taking advantage of the higher redshifts spanned by the DES (and PS1-MDS; Lunnan et al. 2018) events, we investigate possible redshift evolution of the engine and ejecta parameters. We do not find any clear evidence for such evolution, with no significant correlation $(|\rho| \lesssim 0.36)$ between redshift and any physical parameter when the modeling is done consistently with MOSFiT.

Upcoming optical surveys with greater sensitivity and wider field (i.e., Rubin Observatory and Roman Space Telescope) will discover thousands of SLSNe per year to $z \approx 5$ (Villar et al. 2018). These samples will significantly expand our ability to discern evolution in SLSN properties.

\section{ACKNOWLEDGMENTS}

We thank Rosanne Di Stefano and K. Azalee Bostroem for constructive and helpful comments on an early version of this draft. B. H. thanks Ashley Villar, Sebastian Gomez, and Matt Nicholl for help running MOSFiT, and Peter Blanchard for providing simulation data. The computations presented in this work were performed on the FASRC Cannon cluster supported by the FAS Division of Science Research Computing Group at Harvard University. The Berger Time Domain group is supported part by NSF and NASA grants. 


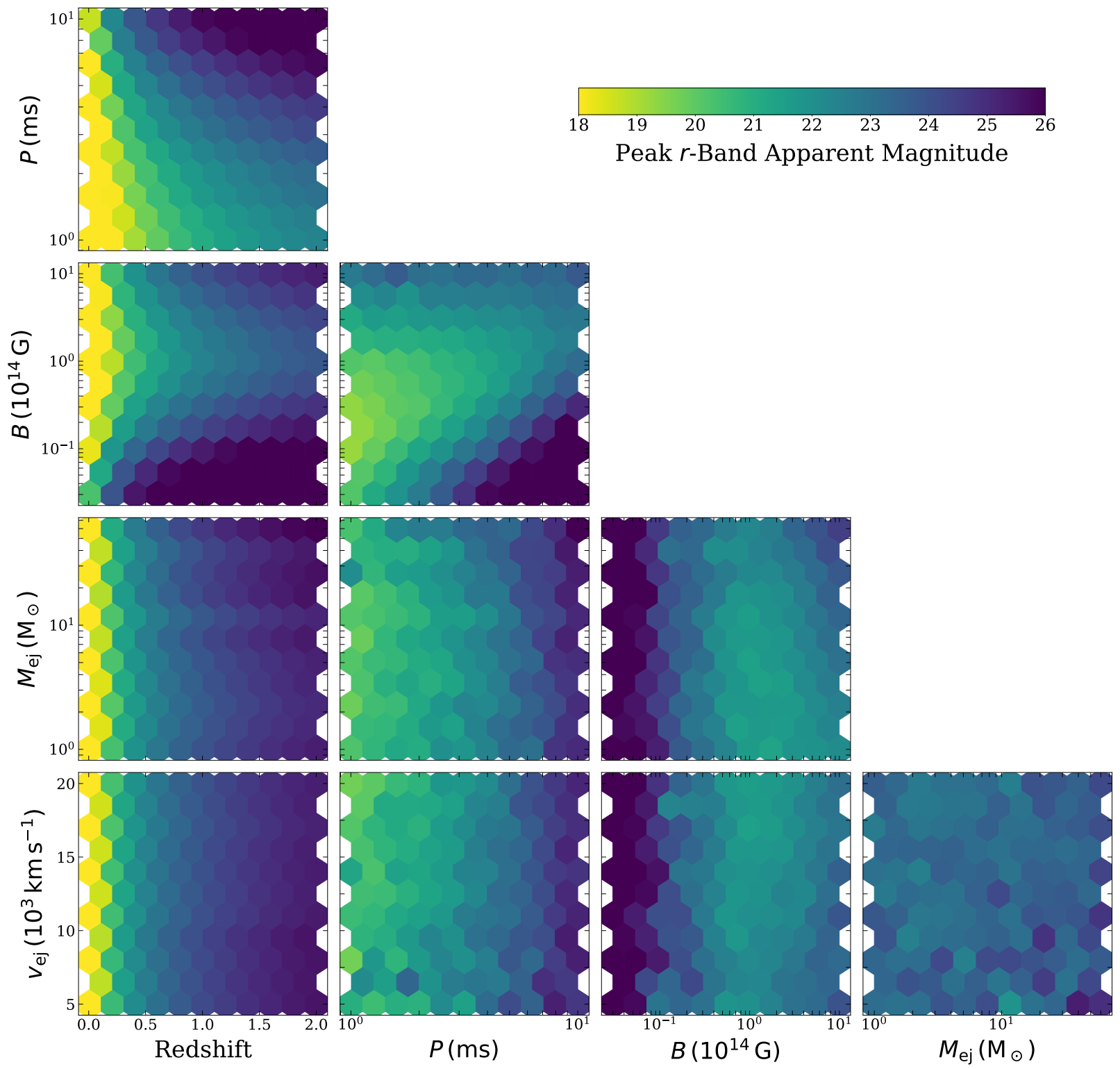

Figure 6. The rest-frame $r$-band apparent magnitude as a function of each pair of physical parameters using 600,000 simulated light curves. The magnitude reported is the sample mean of simulations in each hexagonal cell. The general trend in the $B-P$ space is consistent with observed data, which signifies an observational selection bias. On the other hand, we do not see the strong correlation between $M_{\mathrm{ej}}$ and $P$ as suggested by observed data. We can therefore conclude that the $M_{\mathrm{ej}}-P$ correlation is a physical effect.

Software: Astropy (Astropy Collaboration et al. 2013, 2018), extinction (Barbary 2016), MOSFiT (Guillochon et al. 2018), Matplotlib (Hunter 2007), NumPy (Oliphant 2006), pymccorrelation (Curran 2014; Privon et al. 2020), pyphot, Scipy (Virtanen et al. 2020)

Facility: ADS, DES 


\section{REFERENCES}

Angus, C. R., Smith, M., Sullivan, M., et al. 2019, MNRAS, 487, 2215

Astropy Collaboration, Robitaille, T. P., Tollerud, E. J., et al. 2013, A\&A, 558, A33

Astropy Collaboration, Price-Whelan, A. M., Sipőcz, B. M., et al. 2018, AJ, 156, 123

Barbary, K. 2016, JOSS, 1, 58

Bernstein, J. P., Kessler, R., Kuhlmann, S., et al. 2012, ApJ, 753, 152

Blanchard, P. K., Berger, E., Nicholl, M., \& Villar, V. A. 2020, ApJ, 897, 114

Blanchard, P. K., Nicholl, M., Berger, E., et al. 2019, ApJ, 872, 90

Blanchard, P. K., Nicholl, M., Berger, E., et al. 2018, ApJ, 865, 9

Chomiuk, L., Chornock, R., Soderberg, A. M., et al. 2011, ApJ, 743,114

Curran, P. A. 2014, arXiv:1411.3816

Dark Energy Survey Collaboration. 2005, arXiv:astro-ph/0510346

Dark Energy Survey Collaboration. 2016, MNRAS, 460, 1270

De Cia, A., Gal-Yam, A., Rubin, A., et al. 2018, ApJ, 860, 100

Dessart, L., Hillier, D. J., Waldman, R., Livne, E., \& Blondin, S. 2012, MNRAS, 426, L76

Flaugher, B., Diehl, H. T., Honscheid, K., et al. 2015, AJ, 150, 150

Gal-Yam, A. 2012, Science, 337, 927

Gelman, A., \& Rubin, D. B. 1992, StaSc, 7, 457

Guillochon, J., Nicholl, M., Villar, V. A., et al. 2018, ApJS, 236, 6

Howell, D. A., Sullivan, M., Perrett, K., et al. 2005, ApJ, 634, 1190

Hunter, J. D. 2007, CSE, 9, 90

Inserra, C., Nicholl, M., Chen, T. W., et al. 2017, MNRAS, 468, 4642

Jerkstrand, A., Smartt, S. J., Inserra, C., et al. 2017, ApJ, 835, 13

Kasen, D., \& Bildsten, L. 2010, ApJ, 717, 245

Kasen, D., Metzger, B. D., \& Bildsten, L. 2016, ApJ, 821, 36

Kessler, R., Marriner, J., Childress, M., et al. 2015, AJ, 150, 172

Leloudas, G., Chatzopoulos, E., Dilday, B., et al. 2012, A\&A, 541, A129

Liu, L.-D., Wang, S.-Q., Wang, L.-J., et al. 2017, ApJ, 842, 26

Liu, Y.-Q., Modjaz, M., \& Bianco, F. B. 2017, ApJ, 845, 85

Lunnan, R., Chornock, R., Berger, E., et al. 2013, ApJ, 771, 97
Lunnan, R., Chornock, R., Berger, E., et al. 2014, ApJ, 787, 138

Lunnan, R., Chornock, R., Berger, E., et al. 2018, ApJ, 852, 81

Margalit, B., Metzger, B. D., Thompson, T. A., Nicholl, M., \& Sukhbold, T. 2018, MNRAS, 475, 2659

Mazzali, P. A., Sullivan, M., Pian, E., Greiner, J., \& Kann, D. A. 2016, MNRAS, 458, 3455

Metzger, B. D., Margalit, B., Kasen, D., \& Quataert, E. 2015, MNRAS, 454, 3311

Morganson, E., Gruendl, R. A., Menanteau, F., et al. 2018, PASP, 130, 074501

Nicholl, M., Berger, E., Blanchard, P. K., Gomez, S., \& Chornock, R. 2019, ApJ, 871, 102

Nicholl, M., Berger, E., Margutti, R., et al. 2017a, ApJL, 845, L8

Nicholl, M., Berger, E., Margutti, R., et al. 2017b, ApJL, 835, L8

Nicholl, M., Guillochon, J., \& Berger, E. 2017c, ApJ, 850, 55

Nicholl, M., \& Smartt, S. J. 2016, MNRAS, 457, L79

Nicholl, M., Smartt, S. J., Jerkstrand, A., et al. 2015, MNRAS, 452,3869

Nicholl, M., Berger, E., Smartt, S. J., et al. 2016a, ApJ, 826, 39

Nicholl, M., Berger, E., Margutti, R., et al. 2016b, ApJL, 828, L18

Nicholl, M., Blanchard, P. K., Berger, E., et al. 2018, ApJL, 866, L24

Oliphant, T. E. 2006, A guide to NumPy (USA: Trelgol Publishing)

Perley, D. A., Quimby, R. M., Yan, L., et al. 2016, ApJ, 830, 13

Piro, A. L. 2015, ApJL, 808, L51

Planck Collaboration, Ade, P. A. R., Aghanim, N., et al. 2016, A\&A, 594, A13

Privon, G. C., Ricci, C., Aalto, S., et al. 2020, ApJ, 893, 149

Quimby, R. M., Kulkarni, S. R., Kasliwal, M. M., et al. 2011, Natur, 474, 487

Quimby, R. M., De Cia, A., Gal-Yam, A., et al. 2018, ApJ, 855, 2

Schlafly, E. F., \& Finkbeiner, D. P. 2011, ApJ, 737, 103

Schulze, S., Krühler, T., Leloudas, G., et al. 2018, MNRAS, 473, 1258

Spearman, C. 1904, The American Journal of Psychology, 15, 72

Villar, V. A., Nicholl, M., \& Berger, E. 2018, ApJ, 869, 166

Virtanen, P., Gommers, R., Oliphant, T. E., et al. 2020, NatMe, 17,261

Woosley, S. E. 2010, ApJL, 719, L204

Yan, L., Quimby, R., Gal-Yam, A., et al. 2017, ApJ, 840, 57 